\title{
Pro-Inflammatory Role of Platelets in Hypertension-Mediated End-Organ Damage
}

\author{
Editorial to: "Inhibition of Platelet Activation by Clopidogrel Prevents Hypertension- \\ Induced Cardiac Inflammation and Fibrosis" by L.-X. Jia et al.
}

\author{
Xiao-Jun Du • Helen Kiriazis
}

Published online: 14 August 2013

(C) Springer Science+Business Media New York 2013

Recently, the pro-inflammatory actions of platelets have received increasing attention $[1,2]$. Platelets contribute to inflammation through vesicular release of inflammatory mediators and this function is even more important considering the fact that about $80 \%$ of circulating microparticles are derived from platelets. Another mechanism for the inflammationpromoting action of platelets is to interact with and "inflame" leukocytes [2]. Contribution of platelets to innate immune responses has been well documented in diseased settings such as acute myocardial ischemia and infarction [1,3]. In addition, hypertensive patients are at a higher risk for thrombotic events and show elevated parameters that suggest platelets are activated, including circulating platelet microparticles [4], $\beta$ thromboglobulin [5] and larger platelet size [6]. However, the precise mechanisms responsible for platelet activation in hypertension and its pathogenic role in mediating target organ injury remain unknown.

In this issue, Jia and colleagues [7] report platelet-promoted inflammation and cardiac pathology in a murine model of hypertension induced by angiotension II (Ang II) infusion with a sustained increase in systolic blood pressure from 120 to $150 \mathrm{mmHg}$. While a 7-day period of hypertension did not result in significant cardiac dysfunction, cardiac hypertrophy and perivascular fibrosis were evident by quantitative histology and expression of hypertrophic and fibrotic gene patterns. Using flow cytometry, Jia et al. demonstrated platelet activation and platelet-leukocyte conjugation evoked by Ang II infusion [7]. The causal role of platelet activation in the hypertensive setting and cardiac pathology, measured as cardiomyocyte hypertrophy, fibrosis and inflammatory infiltration, is indicated by the finding that clopidogrel (anti-

\section{X.-J. Du $(\varangle) \cdot$ H. Kiriazis}

Experimental Cardiology Laboratory, Baker IDI Heart and Diabetes Institute, 75 Commercial Road, Melbourne, Victoria 3004, Australia e-mail: xiao-jun.du@bakeridi.edu.au platelet agent) treatment given at the time of Ang II administration largely prevented the cardiac histopathology changes. Thus, as the authors concluded, this study provided a novel mechanism for hypertension-mediated cardiac injury by documenting a pivotal role of platelets in the cardiac histopathology and remodeling.

Demonstration of platelets contributing to cardiac damage following hypertension is of great interest. The pressor action of Ang II was not affected by clopidogrel treatment [7] and hence inhibition by this treatment on cardiac histopathology is not due to a difference in the extent of afterload. Hypertrophic growth and fibrogenesis are expected to be stimulated by regional inflammatory signaling, which is blunted by the anti-platelet treatment, as shown by Jia et al. [7], implying a pro-inflammatory action of platelets. By directly activating Ang II type-1 receptors (AT1), Ang II is known to stimulate cardiomyocyte hypertrophy and fibroblast activation and proliferation, changes that are unlikely to be directly interfered by clopidogrel. Thus, the interplay between platelets and cardiac cells is the likely mechanism contributing to the development of hypertrophy and fibrosis. The authors observed elevated pro-fibrotic signaling measured as elevated transforming growth factor $\beta$ (TGF $\beta)$ at both protein and mRNA levels [7]. Whilst platelets likely release TGF $\beta$ and other growth factors, increased mRNA level of TGF $\beta$ indicates enhanced expression by other cells (such as monocytes and fibroblasts) since platelets are anucleate. Further, considering the fact that the cardiac fibrosis was largely restricted to the perivascular regions, it is also likely that activation of platelets by some as yet undefined mechanisms, promotes a number of bonemarrow derived cells, such as fibrocytes, that eventually migrate into perivascular regions of the heart and differentiate into fibroblasts. Two subtypes of monocytes have been defined as inflammatory (M1) and reparative subtypes (M2) [8]. With the findings by Jia et al. [7] of increased plateletleukocyte interaction and cardiac fibrosis, it would be 
interesting to determine the subtype of monocytes that bind to and are activated by platelets under conditions of hypertension.

The classic mode of platelet activation (Fig. 1a, b) involves platelets interacting with endothelial cells through P-selectin/ P-selectin glycoprotein ligand-1 (PSGL-1) or intercellular adhesion molecule/integrin Mac 1 [9]. Following platelet aggregation and thrombus formation, platelet-rich thrombus interacts with circulating leukocytes promoting leukocyte infiltration into diseased tissues (Fig. 1b). Also, Ang II mediated stimulation of vascular endothelial cells with enhanced expression of adhesion molecules [10] that would be expected to contribute to platelet activation as observed by Jia et al. The findings by Jia and colleagues [7] support an alternative mode by which circulating platelets are activated via an increased shear stress (Fig. 1c). Furthermore, findings by Jia et al. [7] also implicate a hormonal mechanism for activation of circulating platelets. Platelets are equipped with a variety of receptors. Except for platelet-specific receptors, such as P2Y (for ADP), proteinase activated receptors (PAR, for thrombin) and thrombin receptor (for thromboxane A2), platelets are also equipped with other receptors including $\mathrm{AT}_{1}$, Toll-like receptor (TLR, for danger-activated molecular pattern, DAMP, such as histones, fragments of extracellular matrix) and $\alpha_{2}$ adrenoceptor (for catecholamines). There is good evidence that Ang II can directly activate circulating platelets, a possibility for rapid activation of platelets minutes after infusion with Ang II $[11,12]$. In pathological settings, hormonal activation of circulating platelets are likely to play a more important role, as shown recently that circulating histone is potent in activating platelets (Fig. 1d) [13].

The implication by the study by Jia et al. [7] on drug development is obvious: the inflammatory properties of platelets constitutes a potential therapeutic target. The currently available anti-platelet agents, aspirin, clopidogrel, prasugrel, abciximab, and eptifibatide, have been shown to have varying effects on inflammatory markers, particularly those associated with activated platelets such as CD40 ligand, P-selectin, and $\mathrm{C}$-reactive protein. Interestingly, studies have revealed differences in the potency of anti-inflammatory actions among antiplatelet drugs $[14,15]$. In addition, anti-platelet drugs are unavoidably associated with increased risk of bleeding. It would be worthwhile, based on current anti-platelet agents, to develop new drugs with more potent inhibition on platelet anti-inflammatory than hemostatic actions. In this regard, we previously observed that PSGL-1 blocking

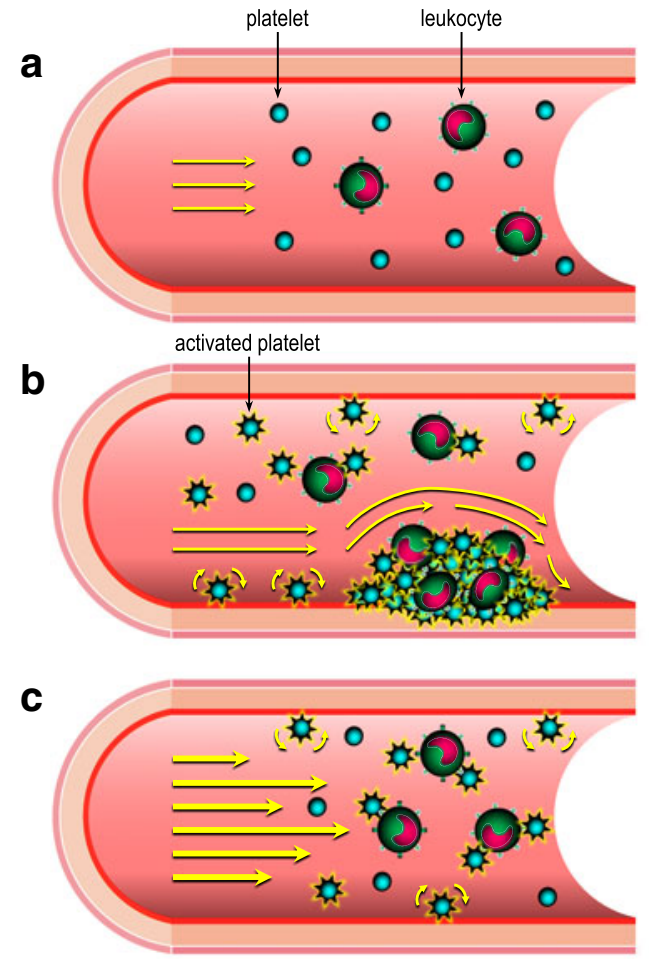

Fig. 1 Schematic diagram depicting different modes by which circulating platelets are activated with subsequent platelet-leukocyte interaction leading to inflammation. a physiological conditions showing circulating non-activated platelets and leukocytes. b the classical mode involves adhesion and aggregation of platelets to vascular sites with endothelial injury or atherosclerotic lesion. c Circulating platelets can be activated by increased shear stress, as indicated by the commented study. d Hormonal

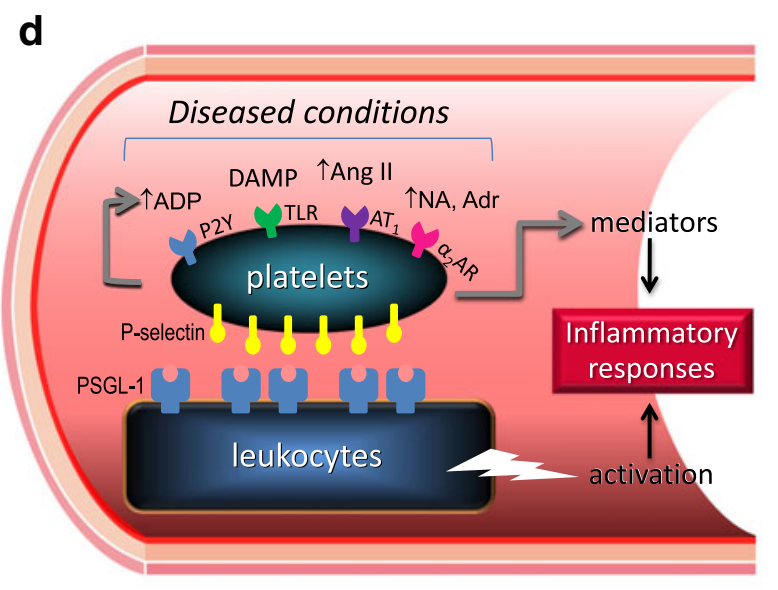

mode represents an alternative mechanism of platelet activation by a group of hormones or molecules that bind to and activate respective receptors at the surface of platelets. $A D P$ adenosine diphosphate, DAMP danger-activated molecular pattern, Ang II angiotensin II, NA noradrenaline, $A d r$ adrenaline, TLR Toll-like receptor, $A T_{1}$ angiotensin II type-1 receptor, $\alpha_{2}-A R \quad \alpha_{2}$-adrenoceptor, PSGL-1 P-selectin glycoprotein ligand-1 
antibody effectively inhibited platelet-leukocyte conjugation [3].

Vascular inflammation has been regarded as a key factor in the pathogenesis of hypertension. Whilst inflammatory cells such as lymphocytes are known to contribute to the pathogenesis of hypertension [16], the role of platelets remains to be examined. The potent inhibition of platelet-leukocyte interaction and cardiac inflammation in the hypertensive model, as shown by Jia et al. [7], raises the potential of using antiplatelet drugs to attenuate hypertension-associated inflammation. It would be of interest to investigate if anti-platelet therapy, given chronically, might limit vascular remodeling and inflammation as well as damage of other organs, particularly the kidney. Indeed, the same group recently showed that clopidogrel treatment attenuated aortic inflammation and progression of aneurysm [17].

Acknowledgment XJD's research was funded by the National Health and Medical Research Council of Australia (1043026).

\section{References}

1. von Hundelshausen $P$, Weber C. Platelets as immune cells: bridging inflammation and cardiovascular disease. Circ Res. 2007;100:27-40.

2. Zarbock A, Polanowska-Grabowska RK, Ley K. Platelet-neutrophilinteractions: linking hemostasis and inflammation. Blood Rev. 2007;21:99-111.

3. Liu Y, Gao XM, Fang L, Jennings NL, Su Y, Xu Q, et al. Novel role of platelets in mediating inflammatory responses and ventricular rupture or remodeling following myocardial infarction. Arterioscler Thromb Vasc Biol. 2011;31:834-41.

4. Preston RA, Jy W, Jimenez JJ, Mauro LM, Horstman LL, Valle M, et al. Effects of severe hypertension on endothelial and platelet microparticles. Hypertension. 2003;41:211-7.

5. Nadar SK, Blann AD, Kamath S, Beevers DG, Lip GY. Platelet indexes in relation to target organ damage in high-risk hypertensive patients: a substudy of the Anglo-Scandinavian Cardiac Outcomes Trial (ASCOT). J Am Coll Cardiol. 2004;44:415-22.

6. Coban E, Yazicioglu G, Berkant Avci A, Akcit F. The mean platelet volume in patients with essential and white coat hypertension. Platelets. 2005;16:435-8.

7. Jia L-X, Qi G-M, Li T-T, Liu O, Yang M, Cui W, et al. Platelet activation is an early event in high blood pressureinduced cardiac inflammation and fibrosis. Cardiovasc Drugs Ther. 2013. doi:10.1007/s10557-013-6471-z

8. Nahrendorf M, Swirski FK. Monocyte and macrophage heterogeneity in the heart. Circ Res. 2013;112:1624-33.

9. Ghasemzadeh M, Kaplan ZS, Alwis I, Schoenwaelder SM, Ashworth $\mathrm{KJ}$, Westein E, et al. The CXCR1/2 ligand NAP-2 promotes directed intravascular leukocyte migration through platelet thrombi. Blood. 2013;121:4555-66.

10. Pueyo ME, Gonzalez W, Nicoletti A, Savoie F, Arnal JF, Michel JB. Angiotensin II stimulates endothelial vascular cell adhesion molecule-1 via nuclear factor- $\mathrm{KB}$ activation induced by intracellular oxidative stress. Arterioscler Thromb Vasc Biol. 2000;20:645-51.

11. Kalinowski L, Matys T, Chabielska E, Buczko W, Malinski T, Angiotensin II AT1 receptor antagonists inhibit platelet adhesion and aggregation by nitric oxide release. Hypertension. 2002; 40:521-7.

12. Schwemmer M, Sommer O, Bassenge E. Angiotensin receptor blocker losartan suppresses platelet activity by interfering with thromboxane signaling. Cardiovasc Drugs Ther. 2001;15:301-7.

13. Semeraro F, Ammollo CT, Morrissey JH, Dale GL, Friese P, Esmon $\mathrm{NL}$, et al. Extracellular histones promote thrombin generation through platelet-dependent mechanisms: involvement of platelet TLR2 and TLR4. Blood. 2011;118:1952-61.

14. Du XJ, Shan L, Gao XM, Kiriazis H, Liu Y, Lobo A, et al. Role of intramural platelet thrombus in the pathogenesis of wall rupture and intra-ventricular thrombosis following acute myocardial infarction. Thromb Haemost. 2011;105:356-64.

15. Pitchford SC. Novel uses for anti-platelet agents as anti-inflammatory drugs. Br J Pharmacol. 2007;152:987-1002.

16. Barhoumi T, Kasal DA, Li MW, Shbat L, Laurant P, Neves $\mathrm{MF}$, et al. T regulatory lymphocytes prevent angiotensin II-induced hypertension and vascular injury. Hypertension. 2011;57:46976.

17. Liu O, Jia L, Liu X, Wang Y, Wang X, Qin Y, et al. Clopidogrel, a platelet P2Y12 receptor inhibitor, reduces vascular inflammation and angiotensin II induced-abdominal aortic aneurysm progression. PLoS One. 2012;7:e51707. 